\title{
Deux estampilles sur tegulae bipedales de la villa de Kervenennec en Pont-Croix (Finistère)
}

Two Stamps on Tegulae Bipedales from the Kervenennec Villa in Pont-Croix (Finistère)

Zwei Ziegelstempel auf tegula bipedales aus der römischen Villenanlage von Kervenennec bei Pont-Croix (Finistère)

Dos tegulae bipedales de la villa de Kervenennec en Pont-Croix (Finistère)

\section{Patrick Galliou}

\section{OpenEdition}

\section{Journals}

Édition électronique

URL : http://journals.openedition.org/rao/2730

DOI : $10.4000 /$ rao. 2730

ISBN : 978-2-7535-4053-8

ISSN : 1775-3732

\section{Éditeur}

Presses universitaires de Rennes

\section{Édition imprimée}

Date de publication : 15 décembre 2014

Pagination : 403-407

ISBN : 978-2-7535-4051-4

ISSN : 0767-709X

Référence électronique

Patrick Galliou, « Deux estampilles sur tegulae bipedales de la villa de Kervenennec en Pont-Croix (Finistère) », Revue archéologique de l'Ouest [En ligne], 31 | 2014, mis en ligne le 15 décembre 2016, consulté le 05 décembre 2020. URL : http://journals.openedition.org/rao/2730 ; DOI : https://doi.org/ $10.4000 /$ rao. 2730 


\title{
Deux estampilles sur tegulae bipedales de la villa de Kervenennec en Pont-Croix (Finistère)
}

\author{
Two Stamps on Tegulae Bipedales from the Kervenennec Villa in Pont-Croix (Finistère)
}

\author{
Patrick GALLIOU*
}

Résumé : Deux grandes briques (tegulae bipedales) estampillées du II ${ }^{\mathrm{e}}$ siècle apr. J.-C., découvertes anciennement dans la villa de Kervenennec en Pont-Croix (Finistère), proviennent incontestablement des figlinae de Rome ou des environs immédiats de l'Urbs. Aucun autre objet de ce type n'étant connu dans l'Ouest de la Gaule, il est probable que ces deux pièces n'aient pas fait l'objet d'un véritable commerce mais aient seulement été intégrées au lest d'un navire avant d'être vendues dans un port voisin aux bâtisseurs de la villa.

\begin{abstract}
Two large $2^{\text {nd }}$ century stamped bricks (tegulae bipedales), found in the 1970s on the site of a Roman villa at Pont-Croix (Finistere), certainly come from the figlinae of Rome itself or of its vicinity. No such similar object having ever been found in Western Gaul, it is very unlikely that they were traded so far north. They were probably included in the ballast of a ship sailing in the Atlantic before being sold in a neighbouring port to the villa builders.
\end{abstract}

Mots clés : Estampilles, tegulae bipedales, villa romaine, Pont-Croix (Finistère), Rome, commerce maritime.

Keywords: Stamps, tegulae bipedales, Roman villa, Pont-Croix (Finistère), Rome, maritime trade.

Lors de l'examen préparatoire à une exposition du mobilier archéologique découvert lors des prospections et fouilles menées sur la villa de Kervenennec en Pont-Croix $(\text { Finistère })^{1}$, nous avons eu la surprise d'y découvrir deux fragments de tegulae bipedales ${ }^{2}$ estampillées. Celles-ci proviennent très vraisemblablement, si l'on en juge à leur cassure, des ramassages opérés dans les vestiges du bâtiment thermal de la villa après sa destruction partielle par un bulldozer, lors de la création d'un chemin d'exploitation, dans le cadre d'une opération de remembrement (Sanquer, 1971, p. 56-63). Ces éléments n'avaient pas été étudiés depuis leur découverte.

1/ Estampille circulaire de $9 \mathrm{~cm}$ de diamètre (fig. 1) sur fragment de tegula bidepalis d'une épaisseur variant de 4,8 et $5 \mathrm{~cm}$. Pâte claire, sans vacuoles. a) Couronne externe : C. AQVILI APRILIS EX PAEDI

b) Couronne interne : CAES BIPIDALE DOLIA

C. Aquili Aprilis ex $\mathrm{p}<\mathrm{r}>\operatorname{aedi}(\mathrm{s})$ Caes(aris) bipedale dolia(re)

La marque est celle d'Aprilis Aquiliae Sozomenae, esclave puis affranchi, apparaissant, après manumission, sous le nom de C. Aquilius Aprilis sur les estampilles Dressel no 358-362 (Helen, 1975, p. 105) des années 123-125, époque où il travaillait comme officinator aux figlinae Oceanae (Bodel, 1983, p. 30, n 32), sans doute situées dans la haute vallée du Tibre, près d'Orte. Cette estampille est attestée à Rome (Via Nova, Forum et Panthéon) (CIL XV/1, n³ 362). Une marque de C. Aquili Aprilis est aussi connue à Tarragone (Rico, 1995, p. 781).

* Professeur émérite à l'université de Bretagne occidentale (Brest) - Centre de recherche bretonne et celtique. (p.galliou@wanadoo.fr)

1. Sur ce site, voir en dernier lieu P. Galliou (2010), p. 347-362. Je remercie $M^{\mathrm{me}}$ S. Casadebaig, archéologue du département du Finistère, de m’avoir permis d'accéder à ces deux pièces.

2. Ces éléments d'argile cuite faisant partie de la suspensura d'un hypocauste, ici incomplets, étaient de forme carrée et mesuraient deux pieds de côté (59,2 cm x 59,2 cm); on utilisait parfois aussi des éléments du même type mais de taille inférieure (sesquipedales d'un pied et demi = 44,4 cm de côté). 


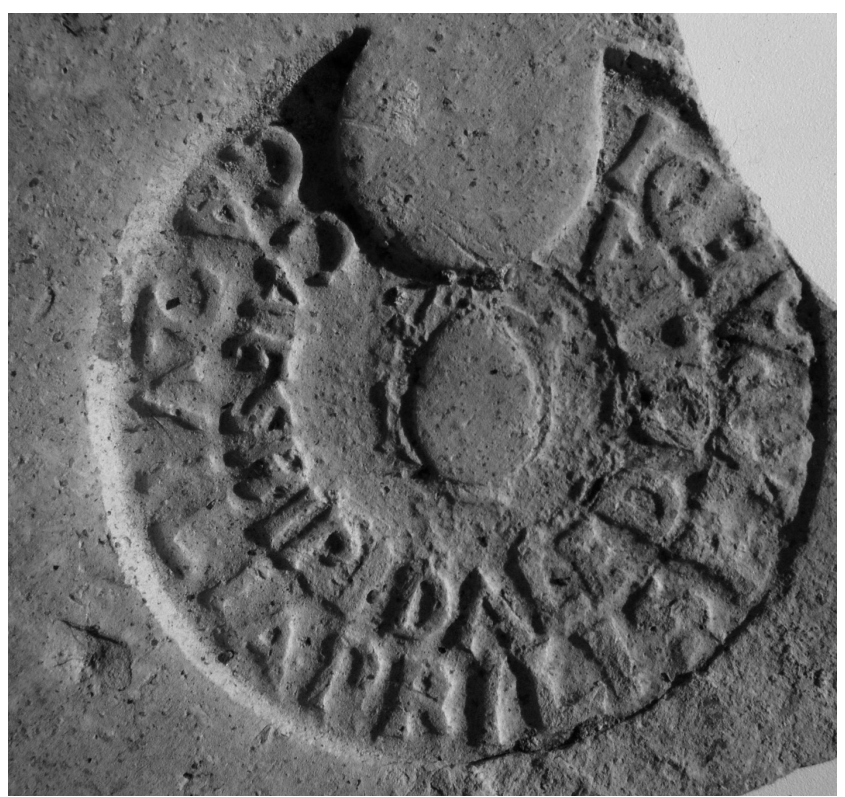

Figure 1 : Kervenennec en Pont-Croix (Finistère) : estampille de C. Aquilius Aprilis. Cliché de l'auteur.

Figure 1: Kervenennec en Pont-Croix (Finistère): stamp of C. Aquilius Aprilis.

2/ Estampille circulaire de 9,7 cm de diamètre (fig. 2) sur fragment de tegula bidepalis, dont l'épaisseur varie de 4 à $4,2 \mathrm{~cm}$. Pâte de couleur crème, à nombreuses vacuoles.

a) Couronne extérieure : EX FIG D D ANNO VERO III [au-dessus] E AMBI

b) Couronne intérieure : $\mathrm{COS}$

Ex fig(linis) Domitiae Domitiorum Ann $<\mathrm{i}>\mathrm{o}$ Vero e(t) Ambi(bulo) co(n)sulibus

Attestée à Ostie (CIL XV/1, n $\left.{ }^{0} 554\right)$, cette estampille est bien datée, Marcus Annius Verus (consul pour la troisième fois) et Caius Eggius Ambibulus étant les consuls ordinaires de l'année 126. La domina est ici Domitia Longina, veuve de Domitien (Chausson, 2003, p. 124, en particulier), et l'atelier sans doute les figlinae Sulpiciana, situées dans les environs des thermes de Caracalla ${ }^{3}$.

La nature, la forme et le contenu de ces deux estampilles montrent que les deux tegulae bipedales de Kervenennec proviennent sans aucun doute des très nombreuses figlinae (ou praedia) installées à l'époque impériale à Rome et plus encore en amont de la ville (Sabine et Ombrie surtout), le long du cours supérieur du Tibre et de ses affluents navigables (Treia, Farfa, Anio) (Steinby, 1981; Morel, 1998; Morel, 2001; Graham, 2006) ${ }^{4}$. Il était bien sûr plus simple

3. Je remercie le professeur Shaw Graham, de l'université de Carleton (Ottawa, Canada), pour ces renseignements.

4. Les figlinae Domitianae de la fin du $\mathrm{I}^{\mathrm{er}}$ siècle et de la première moitié du II $^{\mathrm{e}}$ siècle apr. J.-C. étaient toutefois situées dans le quartier du Latran,

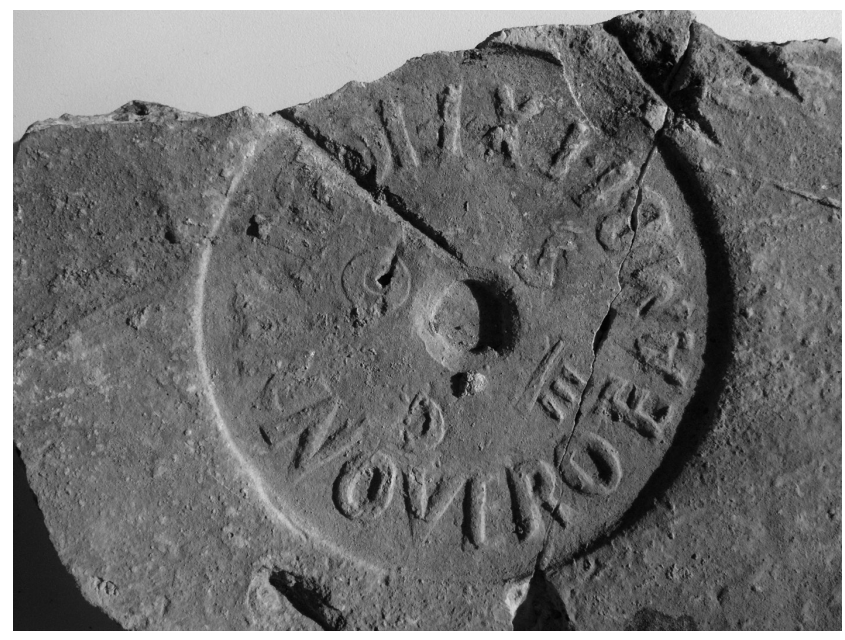

Figure 2 : Kervenennec en Pont-Croix (Finistère) : estampille de Domitia Longina. Cliché de l'auteur.

Figure 2: Kervenennec en Pont-Croix (Finistère): stamp of Domitia Longina.

et moins coûteux de faire descendre les cours d'eau à ces produits pondéreux que de les transporter par voie de terre ou en remontant les fleuves. Les très abondantes marques relevées sur cet opus doliare ont fait l'objet d'études très documentées, dues, en particulier, à l'Institut finlandais de Rome (Descemet, 1880; Bloch, 1947; Helen, 1975; Setälä, 1977; Suolahti, 1977-1978; Bruun, 2005); elles montrent que, jusqu'au début du règne d'Hadrien, les domini, propriétaires de ces figlinae, appartenaient, en majorité, à la classe sénatoriale et que beaucoup d'entre eux étaient des consules ordinarii à consulats souvent multiples 5 . Par la suite, beaucoup de ces unités de production particulièrement rentables ${ }^{6}$ passèrent aux mains des empereurs et de leurs proches ${ }^{7}$, devenant même, à partir du $\mathrm{III}^{\mathrm{e}}$ siècle, un quasi-monopole d'État. Comme le révèlent aussi les estampilles, les officinatores qui géraient ces unités étaient aussi bien des esclaves ou des affranchis, privés ou impériaux, que des hommes - ou des femmes - libres (Weaver, 1998).

La fréquence des dates consulaires que livrent ces marques imprimées dans l'argile permet de juger des crues et décrues de cette industrie : il apparaît ainsi clairement que, née sous la République, celle-ci se développa

qui tire probablement son nom des lateres qu'on y produisait (Santa Maria Scrinari, 1983).

5. On y note aussi la mention de consuls suffects (Bloch, 1944).

6. Bien que plus tardif que nos tegulae, l'Édit du Maximum (301 apr. J.-C.) montre la forte différence entre le salaire des briquetiers et le prix de vente du produit fini (Morel, 1985, p. 100-101; Ferdière, 2012, p. 20).

7. C'est le cas, par exemple, de Domitia Lucilla minor, mère de Marc Aurèle, et de Annia Galeria Faustina, épouse de ce dernier (Chausson, Buonopane, 2010). 
au cours du $\mathrm{I}^{\mathrm{er}}$ siècle apr. J.-C. et connut un très intense floruit pendant les règnes de Trajan (98-117 apr. J.-C.) et surtout de son successeur Hadrien (117-138 apr. J.-C.), au cours desquels les grandes opérations de construction

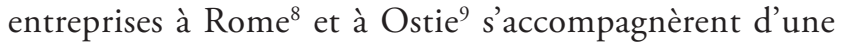
demande considérable de matériaux en terre cuite ${ }^{10}$. On a longtemps supposé, à vrai dire, que ces derniers, façonnés aux environs de Rome, avaient été exclusivement destinés à la consommation architecturale locale, car l'on n'imaginait guère que des éléments aussi ordinaires, qu'il était facile de confectionner à partir de n'importe quelle argile locale et que n'importe quel ouvrier pouvait apprendre à produire en quelques minutes, eussent pu être exportés. On s'aperçoit aujourd'hui qu'il n'en est rien et que les matériaux en terre cuite provenant d'Italie avaient fait l'objet d'un commerce à moyenne et longue distance dans l'Ouest du bassin méditerranéen, et même au-delà de celui-ci (Rico, 1993, fig. 5, en particulier; Rico, 1995). Débarquant à Ostie le vin, le blé ou les céramiques qu'ils avaient chargés en Afrique du Nord, en Tarraconaise, en Sardaigne ou en Gaule narbonnaise, les navires de commerce embarquaient sans doute, comme cargaison ou comme lest (Hartley, 1973), des matériaux de terre cuite, bipedales, sesquipedales et autres, qui, mis à terre dans les ports provinciaux, étaient utilisés dans les programmes de construction locaux, ces importations restant toutefois épisodiques ${ }^{11}$.

En Afrique du Nord, les tegulae estampillées d'origine romaine sont nombreuses à Carthage et, dans une moindre mesure, à Utique, Hadrumète, Rusicade, Saldae et Césarée. La villa de Tagiura, près de Tripoli, en a même livré 78 exemplaires (Zucca, 1987; Tomber, 1987). L'opus doliare urbano se rencontre également en Tarraconaise, à Badalona (Baetulo), Cambrils, Mataró (Iluro), Riudoms, Tarragone (Tarraco), Elche et dans l'île de Majorque (Veny, 1966, 159-160, 162, 166; Steinby, 1981; Rico, 1995, p. 779, 781 ; Piñol, Lopez, Marti, 2000), en Sardaigne (Rico, 1995, p. 779) et enfin en Narbonnaise, sur au moins une dizaine de sites (Glanum [Saint-Rémy-de-Provence, Bouches-du-Rhône] (Rolland, 1944, p. 203), 101.3 (gens Domitia), La Roquebrussane

8. Ainsi les marchés de Trajan (Lancaster, 1998).

9. Trajan avait fait bâtir un nouveau port et des quartiers d'entrepôts, et Hadrien, en tant que duumnvir quinquenal d'Ostie (121 et 126 apr. J.-C.), engagea de grands travaux au centre de la ville (Bloch, 1959; DeLaine, 1996).

10. J.-P. Adam (1984, p. 157) qualifie ainsi Rome d'" univers monumental de briques".

11. C'est l'argument développé par M. Steinby (1981). Ch. Rico (1995, p. 769-777) met aussi en évidence l'importance de la diffusion en Tarraconaise des matériaux en terre cuite provenant de Narbonnaise (Fréjus), que signalent la cargaison de nombreuses épaves et la présence, sur terre, de pièces estampillées provenant des ateliers de Fréjus et de la basse vallée de l'Argens. $(\text { Var })^{12}$, Aix-en-Provence (Bouches-du-Rhône) (CIL XII, 5678, 4 et 9), Rognes (Bouches-du-Rhône) (CIL XII, 5678, 5), L'Almanare (Var) (CIL XII, 5678, 10), Arles (Bouches-duRhône) (CIL XII, 5678, 6), Arnès (Gard) (CIL XII, 5678, 7), Nîmes (Gard) (CIL XII, 5678, 1), Orange (Vaucluse) (CIL XV, 408b, 45), Puyloubier (Bouches-du-Rhône) (CIL XII, 5678, 11), Saint-Jean-de-Garguier (Bouches-du-Rhône) (CIL XII, 5678, 12), Trets (Bouche-du-Rhône) (CIL XII, 5678, 3), soit, pour l'essentiel, à proximité immédiate de Marseille et du delta du Rhône. Au nord de cette zone, le CIL XIII ne mentionne qu'une trouvaille mal localisée en Hollande (CIL XIII, 6, 13097), portant la date consulaire de 123 apr. J.-C., une autre dans le Niederrhein (CIL XIII, 6, 13096), et deux estampilles qui auraient été découvertes dans les jardins du Sénat à Paris (Grivaud de La Vincelle, 1807, p. 163; CIL XIII, 6, 13098-13099).

Les découvertes faites au-delà du bassin méditerranéen sont, on le voit, remarquablement peu nombreuses et la présence de ces deux estampilles urbaines dans la villa de Kervenennec pose, comme l'on s'en doute, de délicats problèmes d'interprétation, d'autant qu'il n'existe, à notre connaissance, aucune autre trouvaille de ce type qui pourrait jalonner l'espace séparant la côte méditerranéenne de la pointe de la péninsule armoricaine ${ }^{13}$. Le très petit nombre de tegulae bipedales estampillées mises au jour à Pont-Croix interdit bien sûr de penser qu'elles aient pu correspondre à une commande passée, pour une raison qui nous échapperait d'ailleurs, à une officine romaine ${ }^{14}$, car il est bien évident qu'elles ne pouvaient suffire, à elles seules, à constituer la suspensura d'une des pièces chaudes du bâtiment thermal. Sans doute est-il alors plus logique de supposer que, chargées sur un navire - ou successivement sur plusieurs navires - avec d'autres matériaux ou denrées - comme lest ou éléments de calage? - elles avaient été débarquées dans les parages de la villa ${ }^{15}$ et achetées par l'entrepreneur chargé de sa transformation ${ }^{16}$. De plus, bien que les civitates armoricaines aient

12. Villa du Grand Loo : Gallia, 39, fasc. 2, 1981, p. 538-539 et fig. 16 (15 tegulae estampillées, datées de 123). Aussi au Sambuc (même site?) : Gallia, 22, 1964, p. 596-597 et fig. 40 (13 tegulae estampillées, datées de 123).

13. Rien n'apparaît ainsi dans le dépouillement très complet effectué par A. Ferdière (2012).

14. À moins, bien sûr, qu’un lot importé ait comporté des tegulae non estampillées et donc non identifiables au premier abord; c'est ainsi le cas à Carthage (site de l'avenue Bourguiba) où l'analyse de la pâte des briques a révélé la présence de briques non marquées importées de la région de Pouzzoles (Fulford, Peacock, 1984, p. 243-244).

15. Peut-être à Pont-Croix, site de fond d'estuaire à marée, à 3 kilomètres à l'est de Kervenennec, qui fut un port fluvial actif jusqu'à l'époque moderne (Gargadennec, 1964, p. 66-67), ou à Audierne, à l'embouchure $\mathrm{du}$ Goyen, où sont connus des ateliers de salaison de poisson d'époque romaine (Galliou, 2010, p. 100-101).

16. L'une des phases de transformation de la villa est attribuée au II siècle (Galliou, 2010, p. 361). 
sans aucun doute importé du vin italien ${ }^{17}$, rien ne permet de penser que le navire en question soit directement venu de la péninsule, car l'on sait que les navires marchands de ce temps pratiquaient souvent le tramping, chargeant et déchargeant au gré des disponibilités qui se présentaient à leur propriétaire ou leur capitaine. Peut-être est-ce d'ailleurs aussi dans le lest de navires fluviaux remontant la Seine et le Rhin que les pièces de Paris, de Hollande et du Niederrhein arrivèrent à leur point de déchargement.

Comme beaucoup de tegulae provenant des figlinae romaines et découvertes hors d'Italie ${ }^{18}$, les deux pièces estampillées de Kervenennec datent des années médianes du règne d'Hadrien, soit ici de 123-125 et 126 apr. J.-C., c'est-à-dire d'une période qui vit se développer considérablement la production des officines romaines et vraisemblablement leur activité d'exportation. Il est bien sûr tentant de penser que ces deux objets peuvent nous permettre de dater avec précision - entre 123 et 126 apr. J.-C. - l'opération de reconstruction des thermes de la villa; il est toutefois prudent de se souvenir, comme l'a bien souligné Évelyne Bukowiecki, que ces matériaux de construction pouvaient être stockés pendant plusieurs mois, sinon plusieurs années, dans des horrea spécialisés avant d'être utilisés dans les chantiers de construction ${ }^{19}$. Si l'on ajoute à ce délai une possible errance de ces pièces à fond de cale d'un navire, il est bien possible qu'elles n'aient pas été intégrées dans les thermes de la villa avant le milieu du $\mathrm{II}^{\mathrm{e}}$ siècle. Malgré leur intérêt, les deux tegulae bipedales de Pont-Croix ne nous renseignent donc qu'à la marge sur le commerce atlantique à l'époque romaine et les opérations de reconstruction de la riche villa de Kervenennec.

\section{Bibliographie}

Adam J.-P., I984 - La Construction romaine. Matériaux et techniques, Paris, Picard.

Bloch H., I 944 - "Consules suffecti on Roman brick stamps ", Classical philology, 39, (4), p. 254-255.

17. En particulier dans des amphores Dressel 2-4, produites, il est vrai dans diverses provinces (Gaule lyonnaise, Tarraconnaise, etc.), mais dont certains exemplaires importés en Armorique paraissent de provenance italienne. Ce type d'amphore fut produit jusqu'au milieu du II $^{\mathrm{e}}$ siècle (Peacock, Williams, 1986, p. 106).

18. L'une des deux tegulae de Paris (CIL XIII, 13098) est cependant datée du règne de Vespasien (= CIL XV, 1. 310). Ch. Rico (1993, p. 55), note que la fréquence avec laquelle apparaît la date consulaire de 123 sur l'opus doliare signifie peut-être que le marquage était devenu une obligation légale cette année-là.

19. É. Bukowiecki (2009) donne un exemple (Palatin, Vigna Barberini) d'un intervalle de trente ans entre la fabrication et l'utilisation d'un lot de bipedales.
BLoch H., I947 - I bolli laterizi e la storia edilizia romana, Rome : Comune di Roma.

Bloch H., I959 - "The Serapeum of Ostia and the brick-stamps of 123 A. D. A new landmark in the history of Roman architecture ", American Journal of Archaeology, 63, (3), p. 225-240.

Bodel J.-P., I983 - Roman Brick Stamps in the Kelsey Museum, Ann Arbor : University of Michigan Press.

Bruun Ch. (dir.), 2005 - Interpretare I bolli laterizi di Roma e della valle del Tevere : Produzione, storia economica, Rome : Institutum Romanum Finlandiae.

Bukowiecki É., 2009 - "Le stockage des briques à Rome ", in Villeneuve F. (éd.), Archéologie de la construction III. Les chantiers de construction en Italie et dans les provinces romaines, Séminaire de l'École normale supérieure, 10-11 décembre 2009 (en ligne, consulté le 15/04/2013).

Chausson F., 2003 - «Domitia Longina : reconsidération d'un destin impérial ", Journal des savants, (1), p. 101-129.

Chausson F. et Buonopane A., 2010 - «Una fonte della ricchezza delle Augustae : Le figlinae urbane ", in Kolb A. (éd.), Augustae, Machtbewusste Frauen am römischen Kaiserhof?Akten der Tagung in Zürich, 18.-20. 09. 2008. Berlin, Akademie Verlag, p. 91-110.

DeLaine J., I 996 - " The Insula of the Paintings. A model for the economics of construction in Hadrianic Ostia ", in Gallina A., Zevi F., Claridge A. (dir.), Roman Ostia Revisited. Archaeological and Historical Papers in Memory of Russell Meiggs. Londres, British School at Rome, p. 165-184.

Descemet Ch., I 880 - Inscriptions doliaires latines. Marques de briques relatives à une partie de la gens Domitia, Paris, Ernest Thorin.

FERDiÈre A., 2OI 2 - "La production de terres cuites architecturales en Gaule et dans l'Occident romain à la lumière de l'exemple de la Lyonnaise et des cités du nord-est de l'Aquitaine : un artisanat rural de caractère domanial? ", Revue archéologique du Centre de la France, 51, p. 17-187.

Fulford M. G. et Peacock D. P. S., I984 - Excavations at Carthage: The British Mission. I. 2. The Avenue du Président Bourguiba. Salammbô. The Pottery and Other Ceramic Objects from the Site, Sheffield, University of Sheffield.

Galliou P., 20 io - Carte archéologique de la Gaule : Le Finistère, Paris, Académie des Inscriptions et Belles-Lettres.

Gargadennec R., I 964 - " Monographie sommaire de PontCroix ", Bulletin de la Société archéologique du Finistère, 90, p. 63-105.

Graham S., 2006 - Ex figlinis: The Network Dynamics of the Tiber Valley Brick Industry in the Hinterland of Rome, Oxford, British Archaeological Reports.

Grivaud de La Vincelle C. M., I 807 - Antiquités gauloises et romaines recueillies dans les jardins du Palais du Sénat pendant les travaux d'embellissement qui y ont été exécutés depuis l'an IX jusqu’à ce jour, Paris, Librairie François-Buisson. 
Hartley K. F., I973 - «La diffusion des mortiers, tuiles et autres produits en provenance des fabriques italiennes ", Cahiers d'archéologie subaquatique, 2, p. 49-60.

Helen T., I975 - Organization of Roman Brick Production in the First and Second Centuries AD, Helsinki, Academia Scientiarum Fennica.

Lancaster L., i 998 - " Building Trajan's Markets ", American Journal of Archaeology, 102, (2), p. 283-308.

Morel J.-P., I 985 - « La manufacture, moyen d'enrichissement dans l'Italie romaine? ", in Leveau Ph. (éd.), L'Origine des richesses dépensées dans la ville antique : sur l'interprétation économique et sociale des données archéologiques, épigraphiques et littéraires en histoire ancienne, Actes du colloque organisé à Aix-en-Provence les 11 et 12 mai 1984, Aix-en-Provence, Université de Provence, p. 87-111.

Morel J.-P., I 998 - " La topographie du commerce et de l'artisanat dans la Rome antique", in L'Urbs. Espace urbain et histoire (Ir siècle av. J.-C.-III siècle apr. J.-C.), Actes du colloque (Rome, 8-12 mai 1985), Rome, École française de Rome : 127-155.

Morel J.-P., 200 I - «Artisanat et manufacture à Rome (I ${ }^{\mathrm{er}}$ s. av. n. è. - $\mathrm{II}^{\mathrm{e}}$ s. de n. è.) ", in La Ville de Rome sous le Haut-Empire : nouvelles connaissances, nouvelles réflexions, Toulouse, université de Toulouse-le-Mirail, (Pallas, n 55), p. 243-263.

Peacock D. P. S. et Williams D. F., I986 - Amphorae and the Roman Economy, Londres, Longman.

Piñol L., Lopez J. et Marti G., 2000 - « Materials ceràmics de construcció de les figlinae urbanes de Roma a Tàrraco ", Empúries, 52, p. 281-288.

Rico Ch., I993 - «Production et diffusion des matériaux de construction en terre cuite dans le monde romain : l'exemple de la Tarraconaise d'après l'épigraphie ", Mélanges de la Casa de Velasquez, 29, (1), p. 51-86.

Rico CH., I995 - "La diffusion par mer des matériaux de construction en terre cuite : un aspect mal connu du com- merce antique en Méditerranée occidentale ", Mélanges de l'École française de Rome. Antiquité, 107, (2), p. 767-800.

Rolland H., I944 - "Inscriptions antiques de Glanum », Gallia, (2), p. 167-223.

SANQUer R., I97 I - "Chronique d'archéologie antique et médiévale. Pont-Croix, Kervenennec ", Bulletin de la Société archéologique du Finistère, 97, p. 56-63.

Santa Maria Scrinari V., I 983 - " Il Laterano e le fornaci di epoca imperiale ", in De Fine Licht K. (dir.), Città e architettura nella Roma imperiale, Odense, Odense University Press, p. 203-218 (Analecta Romana Instituti Danici, Supplementum X).

SetäLÄ P., I 977 - Private Domini in Roman Brick Stamps of the Empire : A Historical Prosopographical Study of Landowners in the District of Rome, Helsinki, Suomalainen Tiedeakatemia.

Steinby M., I98 I - "La diffusione dell'opus doliare urbano ", in Giardina A. et Schiavone A. (dir.), Società romana e produzione schiavistica, III, Bari, Laterza, p. 237-245.

Suolahti J. (dir.), I977-1978 - Lateres signati Ostienses, Rome, Institutum Romanum Finlandiae.

Tomber R., I987 - "Evidence for long-distance commerce. Imported bricks and tiles at Carthage ", Rei Cretariae Romanae Fautorum Acta, 25-26, p. 161-74.

VENY C., I 966 - "Algunas marcas de ladrillos y tejas encontradas en Mallorca ", Archivo español de arqueologia, 39, p. 156-166.

Weaver P., I998 - "Imperial slaves and freedmen in the brick industry ", Zeitschrift für Papyrologie und Epigrafik, 122, p. 238-246.

Zucca R., I 987 - "L'opus doliare urbano in Africa e in Sardinia ", Mastino A. (éd.), L'Africa romana. Atti del IV Convegno di studio (Sassari, 12-14 dicembre 1986), Sassari, Dipartimento di Storia dell'Università di Sassari, p. 659-677.

\footnotetext{
Zusammenfassung: Zwei Ziegelstempel auf tegula bipedales aus der römischen Villenanlage von Kervenennec bei Pont-Croix (Finistère) Zwei große römische Dachziegel (tegulae bipedales) mit Stempelmarken des 2. Jh. n. Ch., die in den 1970er Jahren im Bereich der römischen Villenanlage von Kervenennec bei Pont-Croix (Finistère) gefunden wurden, stammen unzweifelhaft von Ziegeleien (figlinae) aus Rom oder aus dem direkten Umland der urbs. Da aus dem Westen Galliens keine weiteren vergleichbaren Funde vorliegen, müssen wir annehmen, dass diese Ziegel nicht dem regulären Handel zuzuordnen sind, sondern vermutlich als Ballast auf eines der Schiffe gelangt sind, um schließlich in einem benachbarten Hafen an die Erbauer der Villenanlage verkauft zu werden.
}

Resumen : Dos tegulae bipedales de la villa de Kervenennec en Pont-Croix (Finistère) - Dos grandes ladrillos (tegulae bipedales) fechados del siglo II después de Jesús-Cristo, descubiertos en la villa antigua de Kervenennec en Pont-Croix (Finistère), vienen sin duda alguna de las figlinae de Roma o de las afueras inmediatos del Urbs. Ningún otro objeto de este tipo está conocido en el oeste de Galia, asi que es probable que los ladrillos no hayan sido objeto de cualquier comercio real, pero que fueron incluidos al lastre de un buque antes de ser vendidos en un puerto cercano a los constructores de la casa.

Schlüsselwörter: Ziegelstempel, tegulae bipedales, Römische Villenanlage, Pont-Croix (Finistère), Rom, Seehandel.

Palabras clave: tegulae bipedales, villa romaine, Pont-Croix (Finistère), Roma, commercio maritimo. 